[6] R. Siegwart, I. Nourbakhsh Introduction to Autonomous Mobile Robots. Ed. Massachusetts: A Bradford Book, The MIT Press, 2004.

\title{
Література
}

[1] Батанов А.Ф. Робототехнические комплексы для обеспечения специальных операций / Батанов А.Ф., Грицинин С.Н., Муркин С.В. - М.: Специальная техника, 1999, по. 6.

[2] Бурдаков С.Ф. Системы управления движением колесных роботов / Бурдаков С.Ф., Мирошник И.В.,Стельмаков P.Е. - СПб.: Наука, 2001, 229 с.

[3] Ярушкина Н.Г. Основы теории нечетких и гибридных систем. - М.: Финансы и статистика, 2004,320 с.

[4] Юревич Е.И. Основы робототехники, 2-е изд. - СПб.: БХВ-Петербург, 2005, 416 с.

[5] Gacovski Z. Mobile robots - current trends, - Croatia: InTech Janeza, 2011.

[6] Siegwart R. Introduction to Autonomous Mobile Robots / Siegwart R., Nourbakhsh I. - Massachusetts: A Bradford Book, The MIT Press, 2004.

\section{АВТОМАТИЗАЦИЯ РАСПРЕДЕЛЕНИЯ НАГРУЗКИ МЕЖДУ ПАРАЛЛЕЛЬНО РАБОТАЮЩИМИ КОТЛАМИ}

\author{
А. Лысюк ${ }^{1}$ К. Беглов ${ }^{2}$ \\ 1,2Одесский национальный политехнический университет \\ ORCID: ${ }^{1} 0000-0002-4438-673 X ;{ }^{2} 0000-0002-5277-2577$ \\ E-mail: 1'lysyuk92@gmail.com; ${ }^{2}$ beglov.kv@opu.ua
}

Copyright (C 2017 by author and the journal "Automation technological and business - processes". This work is licensed under the Creative Commons Attribution International License (CC BY). http://creativecommons.org/licenses/by/4.0/
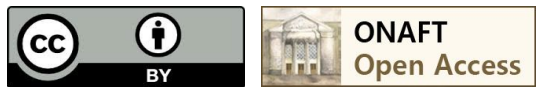

Анотаичя: Рассмотрен синтез поисковой процедуры для оптимизации распределения нагрузки между параллельно работающими котлами в режиме реального времени. Предложен критерий оптимальности работь котла, учитьвающий не только коэффициент полезного действия, но и величину износа оборудования. Решения основань на использовании поискового алгоритма деформируемого симплекса.

Abstract: The synthesis of the search procedure for optimizing load distribution between parallel running boilers in real time mode is considered. A criterion for the optimality of the boiler operation is proposed, taking into account not only the efficiency, but also the wear and tear of the equipment. The solutions are based on the search algorithm of the deformed simplex.

Ключевые слова: автоматическая система управления, котел, нагрузка, оптимизация, оперативное управление.

Keywords: automatic control system, boiler, load, optimization, operational control.

\section{1. Введение}

Классическим методом распределения нагрузки между котлами, работающими на общего потребителя, является распределение по наименьшему относительному приросту расхода топлива. [1]. В виду своей классичности, данный метод применяют на многих энергогенерирующих предприятиях (котельных, ТЭЦ, ТЭС) модифицированный для реализации в современных АСУ ТП.

Однако при оперативном управлении котельным оборудованием, например при возмущениях, связанных с изменением состава топлива, оптимизация его работы не производится. Одной из причин этого является тот факт, что 
поисковые процедуры основаны на расчетных зависимостях удельного расхода топлива от нагрузки котла.

При отклонении состава топлива от расчетного получить указанные зависимости не представляется возможным. Поэтому предлагается разработать алгоритм поиска оптимального распределения нагрузки между параллельно работающими котлами и сжигающими не сертифицированное топливо.

2. Анализ литературных данных и постановка проблемы

Несмотря на то, что вопрос оптимизации распределения нагрузки рассматривается на протяжении десятков лет, вновь и вновь появляются работы, позволяющие повысить качество решений по автоматизированному управлению оборудованием. К таким работам можно отнести $[2,3,4]$.

В статье [2] описывается программный комплекс, позволяющий распределить тепловую и электрическую нагрузку между энергоблоками. Критерием оптимизации является минимум расхода топлива по электростанции в целом. Программный комплекс реализован на базе современных вычислительных средств и SCADA системы. Несомненным достоинством результатов этой работы является уменьшение психофизической нагрузки на операторов при изменении режимов работы основного оборудования, т. к. определение необходимых уровней нагрузки агрегатов возложено на ЭВМ.

В работе [3] приводится подход для определения часового расхода топлива на основе исходных данных относительных приростов расхода топлива блока с применением метода численного интегрирования. В качестве критерия оптимальности указано равенство относительных приростов расхода топлива для котлов, работающих на разных мощностях. Решение задачи оптимального распределения нагрузки решается с помощью пакета Маtlab.

Работа [4] посвящена рассмотрению основных проблем и функциональных возможностей реализации алгоритмов оптимального управления технологическими и производственными процессами блочного и станционного уровней. Также в этой работе отмечено, что с точки зрения управления экономичностью технологического процесса минимизация относительного прироста расхода топлива $\mathrm{b}(\mathrm{t})$ некорректна, так как текущий расход топлива $\mathrm{B}(\mathrm{t})$ из-за инерционности процесса «котел - турбина - генератор» обусловлен не выработкой мощности $\mathrm{N}(\mathrm{t})$, а получением мощности $\mathrm{N}(\mathrm{t}+\tau 3)$, где $\tau 3$ - запаздывание технологического процесса. Применение этого критерия правомерно только для строго стационарных режимов работы оборудования без каких-либо внешних и внутренних возмущений. В переходных и нестационарных режимах удельный расход топлива произвольный и не отражает текущую экономичность работы оборудования.

В последнее время износ оборудования достиг таких значений, что стали появляться работы, в которых обосновывается необходимость учета надежности оборудования. Например в [5] обосновывается введение комплексного критерия оценки работы ТЭС. Среди составляющих такого критерия предлагается учитывать штрафы за выбросы, ухудшающие экологическую обстановку, изменение состава топлива, особенности компоновки оборудования электростанции, состояние основного оборудования с точки зрения надежности. Так как в странах бывшего СССР большинство энергогенерирующих предприятий было введено в эксплуатацию в 1950-е - 1970-е годы, фактор надежности приобретает все большее значение.

Хотя, надо отметить, что вопросам распределения нагрузки с учетом надежности и экологической обстановки уделялось внимание и ранее [6, 7].

Таким образом, на основании вышесказанного, можно констатировать, что вопрос оперативного управления оптимальным распределением нагрузки между параллельно работающими котлами с учетом их надежности до конца не решен.

3. Цель и задачи исследования

Целью работы является разработка алгоритма расчета задающих воздействий регуляторам нагрузки для группы котлов, работающих параллельно. Такой алгоритм должен обеспечить оптимальное распределение нагрузки между котлами с учетом не только коэффициента полезного действия, но и величины износа каждого котла.

Для достижения намеченной цели были выделены следующие задачи:

- определить целевую функцию, оптимизирующую работу котлов;

- определить ограничения, накладываемые на переменные, входящие в целевую функцию;

- разработать процедуру решения оптимизационной задачи;

- проверить адекватность решений, полученных в результате выполнения поисковой процедуры.

4. Разработка алгоритма расчета задающих воздействий

4.1 Определение критерия оптимальности работы котла

В рассматриваемой работе для определения оптимальной нагрузки каждого котла предлагается использовать критерий, учитывающий как его кпд, так и величину износа этого котла. А целевой функцией управления является минимизация суммы критериев оптимальности работы каждого котла.

В работе [8] уже рассматривалась похожая задача. Однако предложенные в указанной работе величины, входящие

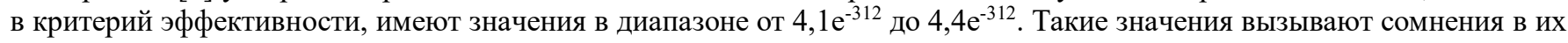
применимости.

В данной работе предлагается использовать значения кпд котла и коэффициент износа оборудования.

Износ оборудования можно оценить коэффициентом износа по аналогии с расчетом величины амортизационных отчислений [9] : 


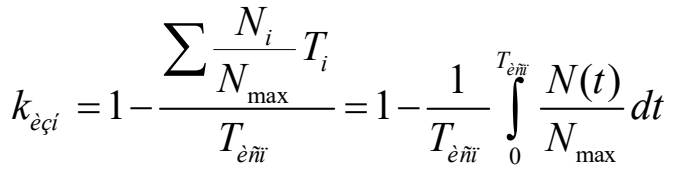

где $\mathrm{Nmax}$ - максимальная нагрузка (мощность) котла;

$\mathrm{Ni}$ - частичная нагрузка (мощность) котла;

Тисп - плановое время эксплуатации котла;

Ti - длительность времени работы котла на частичной нагрузке;

i - количество промежутков времени, при которых котел работает при постоянной нагрузке.

Введем обозначение $\frac{N_{i}}{N_{\max }}=v_{i}$ - относительная мощность котла, $\frac{T_{i}}{T_{\grave{e ̀ n}}}=\tau_{i}$ - относительное время работы котла на частичной нагрузке.

Тогда коэффициент износа можно записать в следующем виде:

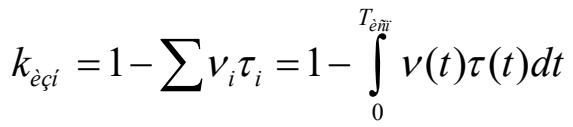

Для текущего момента эксплуатации $\tau=c o n s t$, при этом $0<\tau \leq 1$, поэтому (2) можно записать

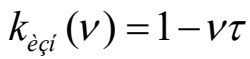

То есть, для текущего момента времени коэффициент износа является функцией мощности.

Зависимость кпд котла от его нагрузки может быть описана квадратичной зависимостью [10]

$$
\eta(N)=c_{1}\left(N-N_{\max }\right)^{2}+c_{2}
$$

где $\mathrm{c}_{1}$ и $\mathrm{c}_{2}-$ константы.

Определим значение с $c_{1}$ и с $c_{2}$ из условий: $\eta\left(N_{\max }\right)=\eta_{0}$ - при максимальной нагрузке кпд равен максимальному (расчетному) значению, - при нулевой нагрузке кпд также равен нулю.

Составив и решив систему уравнений, получим значения коэффициентов: $c_{1}=\frac{\eta_{0}}{N_{\max }^{2}} ; c_{2}=\eta_{0}$

Тогда (3) можно переписать в виде:

$$
\eta(N)=\frac{\eta_{0}}{N_{\max }^{2}}\left(N-N_{\max }\right)^{2}+\eta_{0}
$$

или

$$
\eta(v)=\eta_{0}\left(2 v-v^{2}\right)
$$

Предлагается в качестве целевой функции работы группы котлов использовать выражение:

$$
I\left(\eta, k_{\dot{e} \varsigma i}\right)=\sum_{j=1}^{n}\left(a_{1 j} \eta\left(v_{j}\right)+a_{2 j} k_{\dot{e} \varsigma i}\left(v_{j}\right)\right)
$$

где $\mathrm{n}$ - количество котлов,

$\mathrm{a}_{1 \mathrm{j}}$ и $\mathrm{a}_{2 \mathrm{j}}$ - весовые коэффициенты для ј-го котла.

Преимущество использования выражения (7) по сравнению с другими $[1,2,3,8]$ состоит в следующем:

- величины $\eta(v)$ и $k_{\grave{e ̨ c ̧ i ~}}(v)$ одного порядка, изменяющиеся в диапазоне от 0 до 1 ;

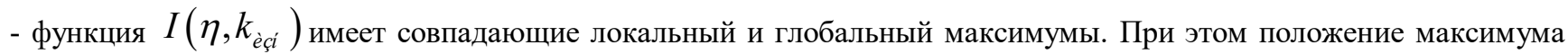
пропорционально величине износа.

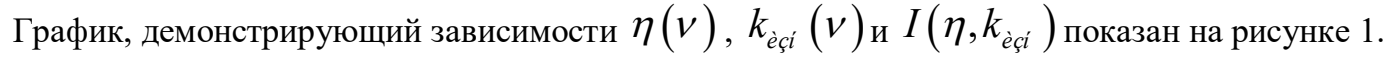

Графики, приведённые на рисунке, построены при использовании следующих допущений: $\eta_{0}=0.9$,

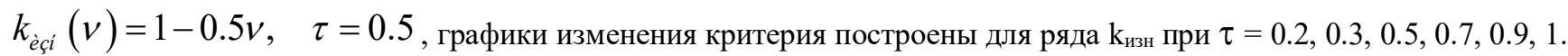

Решение задачи оптимального распределения нагрузки между работающими котлами сводится к поиску максимума целевой функции (7). 


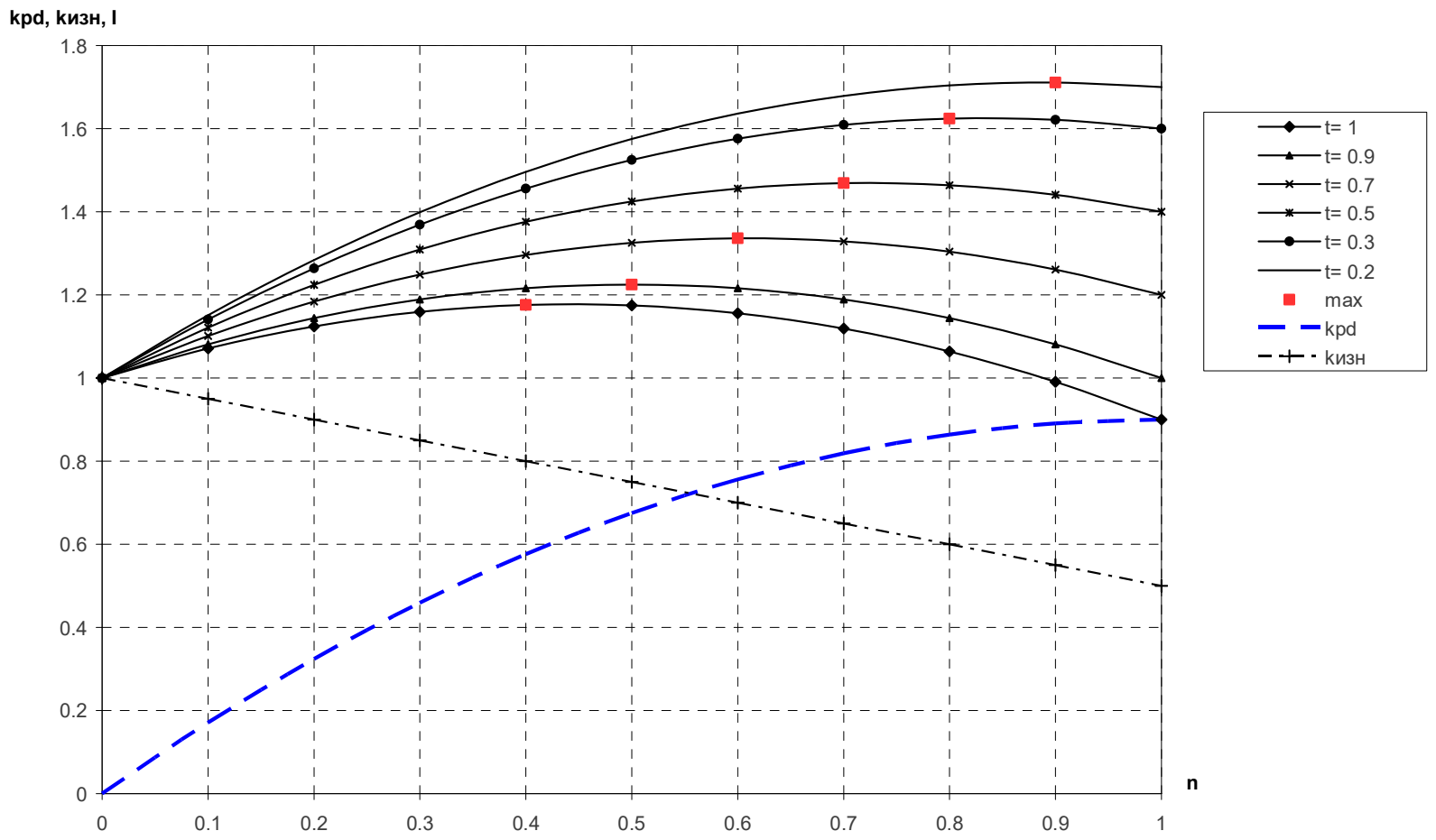

Рис. 1 - Зависимость $\eta, k_{\grave{e} ̧ ̧ i}$ и $I$ от относительной нагрузки $v$

\section{2 Определение ограничений на переменные, входящие в целевую функцию}

На переменные и решения накладываются следующие ограничения и принимаются следующие допущения:

- оборудование (котлы) являются работающим, поэтому решение задачи оптимизации должно проводиться в режиме реального масштаба времени;

- так как оптимизация проводится для реального оборудования, функциональная зависимость кпд от мощности не задана. КПД рассчитывается в режиме реального времени по измеренным значениям технологических параметров;

- значение кпд может изменяться вследствие изменения состава топлива;

- варьируемыми переменными являются заданные значения мощности каждого котла $v_{j}$;

- значение переменной может находиться в диапазоне

$$
0 \leq v_{i} \leq 1
$$

- сумма мощностей всех котлов должна быть равна заданному значению суммарной мощности:

$$
\sum_{j=1}^{n} v_{j}=N_{Z}
$$

\section{3 Разработка процедуры решения оптимизационной задачи}

Для реализации поисковой процедуры предлагается использовать модифицированный симплекс метод (Нелдера Мида), т.к. он не требует нахождения производной минимизируемой функции. Несмотря на то, что указанный метод поиска является безусловным, ограничения (8) легко реализуются программно. А именно, при выходе варьируемой переменной за заданные границы, ей принудительно присваивается граничное значение.

Выражение (9) позволяет уменьшить размерность решаемой задачи на единицу. Т.е. вместо поиска минимума функции $I\left(\eta, k_{\grave{e} i^{i}}\right)$ от $n$ переменных находить минимум функции $\mathrm{I}_{1}$ от $\mathrm{n}-1$ переменной, а после восстановить (вычислить) значения исходных переменных.

Рассмотрим решение задачи оптимизации для работы трех котлов с относительными нагрузками $v_{1}, v_{2}, v_{3}$.

Если представить распределение нагрузки в виде фазового пространства с координатами $v_{1}, v_{2}, v_{3}$, показанное на рисунке 2, то выражение (9) является уравнением плоскости, в пределах которой будет производиться поиск экстремума функции (7).

Следует отметить, что для переменных $v_{1}, v_{2}, v_{3}$ имеется ограничение $v_{j \min } \leq v_{j} \leq 1$. Это ограничение 
обусловлено в первую очередь физической невозможностью работы котла на нагрузках ниже некоторого минимального значения, обычно равного 30\% от максимального значения. Во-вторых, для выполнения условия (9) при работе двух из трех котлов на полной мощности, т.е. при $v=1$, третий котел должен работать с минимальной нагрузкой

$$
v_{3 \min }=N_{Z}-v_{1}-v_{2}=N_{Z}-1-1=N_{Z}-2
$$

Отсюда следует, что если NZ может быть обеспечено работой только двух котлов, то $v_{j \min } \leq 0$. Этот факт можно использовать как условие для отключения одного из котлов системой автоматического управления.

Таким образом, задав ограничения (9) и (10) мы переходим к поиску оптимума на плоскости, проходящей через точки $\mathrm{A}\left(v_{1} \min , 1,1\right), \mathrm{B}\left(1,1, v_{3} \min \right), \mathrm{C}\left(1, v_{2} \min , 1\right)$. Примем, что котлы имеют одинаковое ограничение на минимальную мощность: $v_{1 \min }=v_{2 \min }=v_{3 \min }=v_{\min }$.

Обозначим оси полученной плоскости как $\mathrm{x}_{1}$ и х $\mathrm{x}_{2}$. Начало координат совпадает с точкой А. Положение точки на плоскости определяется двумя координатами. В качестве таких координат выберем расстояния от искомой точки Z( ${ }_{1}$, $\left.\mathrm{x}_{2}\right)$ до точки $\mathrm{A}(0,0)$ и до точки $\mathrm{C}\left(\mathrm{x}_{1 \mathrm{C}}, 0\right)$. Тогда точка $\mathrm{B}$ на плоскости будет иметь координаты $\left(\mathrm{x}_{1 \mathrm{~B}}, \mathrm{x}_{2 \mathrm{~B}}\right)$.

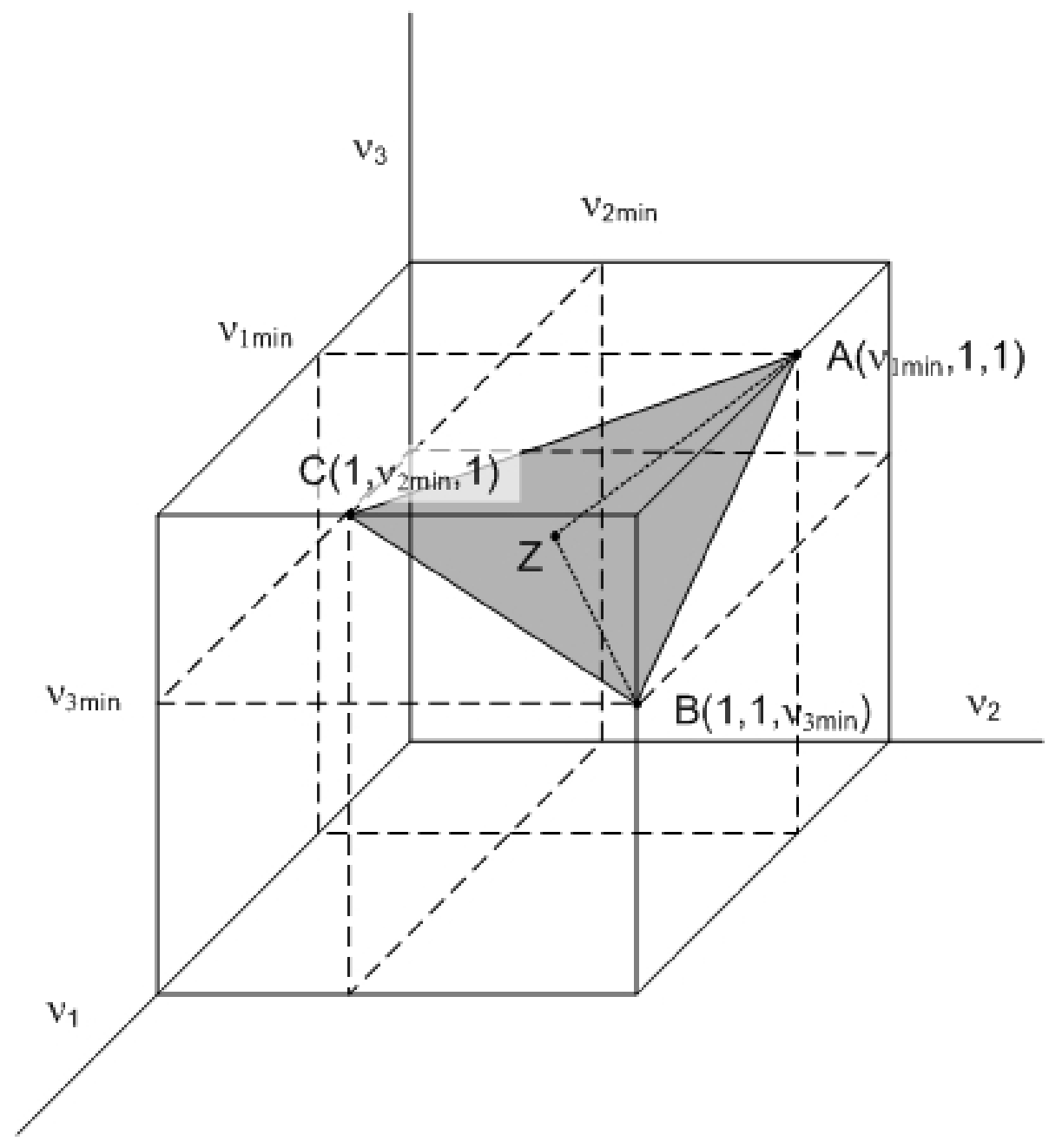

Рис. 2 - Фазовое пространство в координатах относительных мощностей

Взаимное преобразование координат $\left(v_{1}, v_{2}, v_{3}\right)$ и $\left(\mathrm{x}_{1}, \mathrm{x}_{2}\right)$ выполняется на основе равенства расстояний между точками в пространстве и на плоскости:

$$
r=\sqrt{x_{1}^{2}+x_{2}^{2}}=\sqrt{v_{1}^{2}+v_{2}^{2}+v_{3}^{2}}
$$

Фазовая плоскость, для которой проводится поиск минимума (максимума) функции (7) показана на рисунке 3. 


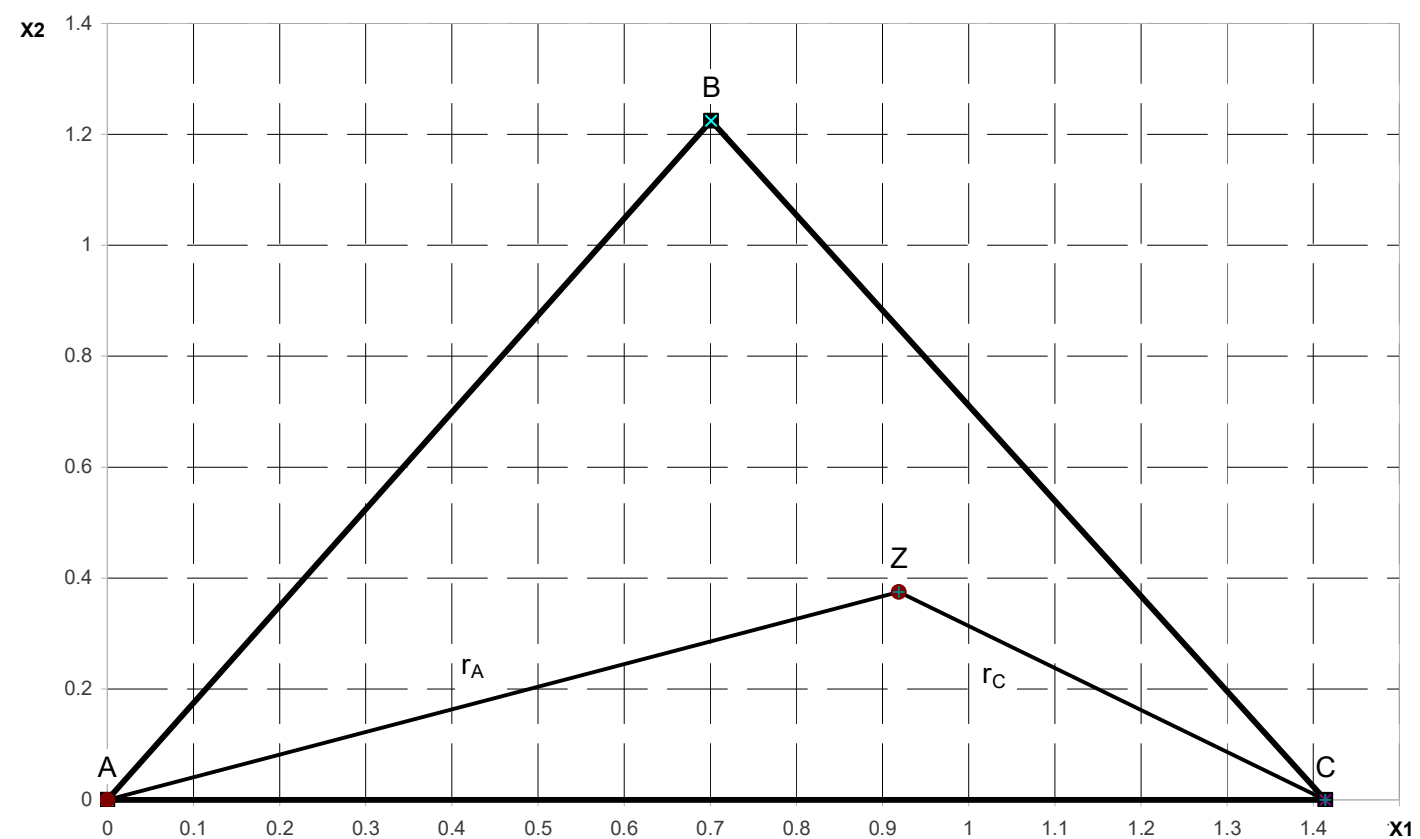

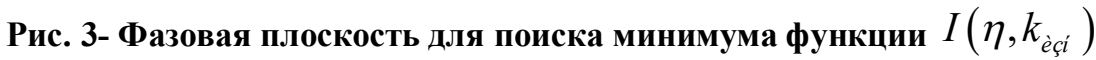

Для проведения процедуры поиска минимума функции $I\left(\eta, k_{\dot{e} \varsigma i}\right)$ в координатах $\left(\mathrm{x}_{1}, \mathrm{x}_{2}\right)$ необходимо ввести дополнительные ограничения (точка минимума должна находиться внугри треугольника $\mathrm{ABC}$ ) :

$$
0 \leq x_{1} \leq x_{1 C} ; x_{2} \geq 0 ; x_{2} \leq \frac{x_{2 B}}{x_{1 B}} x_{1} ; x_{2} \leq \frac{x_{2 B}}{x_{1 B}-x_{1 C}}\left(x_{1}-x_{1 C}\right) ;
$$

Рассчитаем координаты точек $\mathrm{A}, \mathrm{B}$ и С на плоскости исходя из значений $\left(v_{1}, v_{2}, v_{3}\right)$ для этих точек в пространстве:

Координаты точек А, В и С в фазовом пространстве:

$$
\mathrm{A}\left(v_{1 \min }, 1,1\right) ; \mathrm{B}\left(1,1, v_{3 \min }\right) ; \mathrm{C}\left(1, v_{2 \min }, 1\right)
$$

Так как в качестве начала отсчета была выбрана точка $\mathrm{A}$, а направление оси $0 x_{1}$ было выбрано так, чтобы она проходила через точку C, можно записать:

$$
x_{1 A}=0 ; x_{2 A}=0 ; x_{2 C}=0
$$

Для нахождения других координат воспользуемся соотношениями:

$$
\begin{aligned}
& \sqrt{\left(x_{1 A}-x_{1 B}\right)^{2}+\left(x_{2 A}-x_{2 B}\right)^{2}}=\sqrt{\left(v_{1 A}-v_{1 B}\right)^{2}+\left(v_{2 A}-v_{2 B}\right)^{2}+\left(v_{3 A}-v_{3 B}\right)^{2}} \\
& \sqrt{\left(x_{1 C}-x_{1 B}\right)^{2}+\left(x_{2 C}-x_{2 B}\right)^{2}}=\sqrt{\left(v_{1 C}-v_{1 B}\right)^{2}+\left(v_{2 C}-v_{2 B}\right)^{2}+\left(v_{3 C}-v_{3 B}\right)^{2}} \\
& \sqrt{\left(x_{1 C}-x_{1 A}\right)^{2}+\left(x_{2 C}-x_{2 A}\right)^{2}}=\sqrt{\left(v_{1 C}-v_{1 A}\right)^{2}+\left(v_{2 C}-v_{2 A}\right)^{2}+\left(v_{3 C}-v_{3 A}\right)^{2}}
\end{aligned}
$$

Подставляя (13) и (14) в (17) получаем:

$$
\begin{aligned}
& \sqrt{\left(x_{1 C}-0\right)^{2}+(0-0)^{2}}=\sqrt{\left(1-v_{1 \min }\right)^{2}+\left(v_{2 \min }-1\right)^{2}+(1-1)^{2}} \\
& x_{1 C}=\sqrt{2}\left(1-v_{\min }\right)
\end{aligned}
$$

Подставляя (13) и (14) в (15) получаем:

$$
\begin{aligned}
& \sqrt{\left(0-x_{1 B}\right)^{2}+\left(0-x_{2 B}\right)^{2}}=\sqrt{\left(v_{1 \min }-1\right)^{2}+(1-1)^{2}+\left(1-v_{3 \min }\right)^{2}} \\
& x_{1 B}^{2}+x_{2 B}^{2}=2\left(1-v_{\min }\right)^{2}
\end{aligned}
$$

Подставляя (13) и (14) в (16) получаем: 


$$
\begin{aligned}
& \sqrt{\left(x_{1 C}-x_{1 B}\right)^{2}+\left(0-x_{2 B}\right)^{2}}=\sqrt{(1-1)^{2}+\left(v_{2 \min }-1\right)^{2}+\left(1-v_{3 \min }\right)^{2}} \\
& \left(x_{1 C}-x_{1 B}\right)^{2}+x_{2 B}^{2}=2\left(1-v_{\text {min }}\right)^{2}
\end{aligned}
$$

Решая совместно уравнения (19) и (20) с учетом (18) получаем

$$
x_{1 B}=\frac{\sqrt{2}}{2}\left(1-v_{\min }\right) ; x_{2 B}=\sqrt{1.5}\left(1-v_{\min }\right) ;
$$

После нахождения координат точек А, В, С вычисляются ограничения (12) и производится поиск минимума

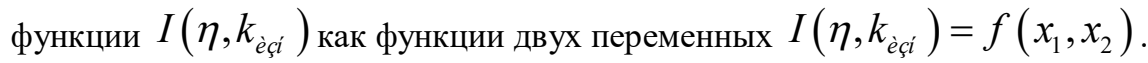

При этом на каждой итерации поиска необходимо производить вычисление значений $\left(v_{1}, v_{2}, v_{3}\right)$. Эти значения можно вычислить, решая систему уравнений:

$$
\left\{\begin{array}{l}
\sqrt{\left(x_{1}-x_{1 A}\right)^{2}+\left(x_{2}-x_{2 A}\right)^{2}}=\sqrt{\left(v_{\min }-v_{1}\right)^{2}+\left(1-v_{2}\right)^{2}+\left(1-v_{3}\right)^{2}} \\
\sqrt{\left(x_{1}-x_{1 C}\right)^{2}+\left(x_{2}-x_{2 C}\right)^{2}}=\sqrt{\left(1-v_{1}\right)^{2}+\left(v_{\min }-v_{2}\right)^{2}+\left(1-v_{3}\right)^{2}} \\
v_{1}+v_{2}+v_{3}=N_{Z}
\end{array}\right.
$$

Данная система уравнений решается численным методом.

4.4 Проверка адекватности решений, полученных в результате выполнения поисковой процедуры

Указанный алгоритм расчета оптимальных значений нагрузки котлов был реализован в пакете Маtlab. Причем, работа оборудования с вычислением значений $\eta_{j}, k_{\grave{e} ̧ \varsigma j}$ и $I\left(\eta, k_{\grave{e} c ̧ i}\right)$, моделировалась в пакете Simulink, а вычислительная поисковая процедура выполнялась путем вызова функции из командной строки Маtlab. Таким образом, были учтены динамические свойства котлов и учтены переходные процессы, вызванные изменением заданий регуляторам.

Для проверки решения были приняты следующие исходные данные.

Котел №1 имеет относительное время работы на частичной нагрузке $\tau_{1}=0,8$, кпд котла описывается выражением: $\eta_{1}=0,95-0,5 *(v 1-0,95)^{2}$.

Котел №2 имеет относительное время работы на частичной нагрузке $\tau_{2}=0,7$, кпд котла описывается выражением: $\eta_{2}=0,9-0,5 *(v 2-0,9)^{2}$.

Котел №3 имеет относительное время работы на частичной нагрузке $\tau_{3}=0,6$, кпд котла описывается выражением: $\eta_{3}=0,85-0,5 *(v 3-1,0)^{2}$.

Суммарная заданная нагрузка котлов $\mathrm{Nz}=\mathrm{v}_{1}+\mathrm{v}_{2}+\mathrm{v}_{3}=2,6$.

Графики зависимости коэффициента износа, кпд и целевой функции каждого котла показаны на рисунке 4.

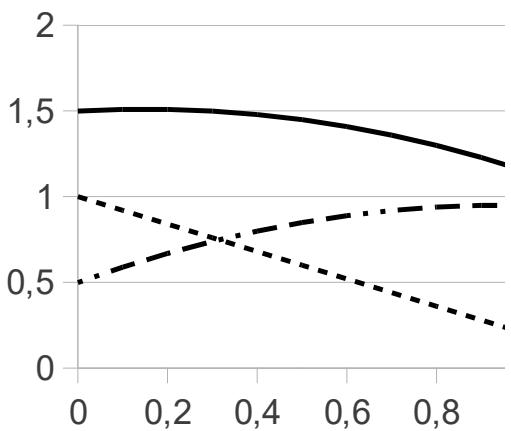

a)

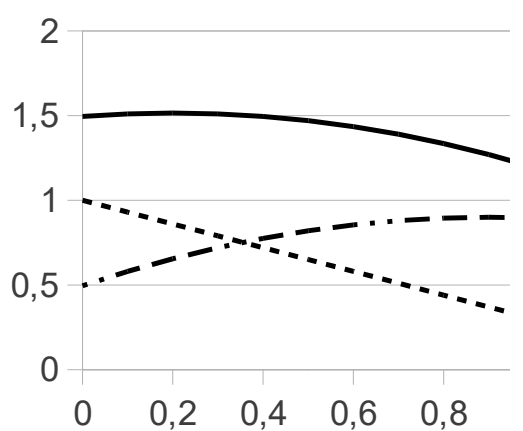

б)

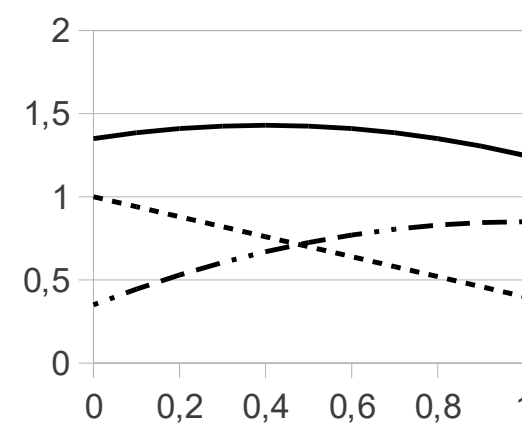

B)

а) котел №1, б) котел №2, в) котел №3

- - целевая функиия, .... - коэффициент износа, ..---.. - кпд

Рис. 4 - Характеристики котлов 
Методом прямого перебора, изменяя относительную мощность $v$ каждого котла, было установлено, что для заданной мощности максимум целевой функции составляет $\mathrm{I}_{\Sigma}=3,8844$. При этом нагрузки котлов составили: $v_{1}=0,775$ $v_{2}=0,825 v_{3}=1$.

Следует отметить, что процедура поиска методом прямого перебора выполнялась около одного часа на ЭВМ с процессором Intel i3-380M с тактовой частотой $2,53 \mathrm{GHz}$. При этом значения относительных мощностей были ограничены диапазоном $v=0,70 \ldots 1$, шаг изменения 0,001 . То есть пришлось рассмотреть $76^{3}=438976$ вариантов. Из них заданной мощности $\mathrm{Nz}=2,6$ удовлетворяли 157 вариантов задания нагрузки.

После этого была запущена процедура поиска оптимального распределения нагрузки с использованием динамической модели котлов. Структурная схема обмена данными между компонентами программного пакета Маtlab показана на рисунке 5 .

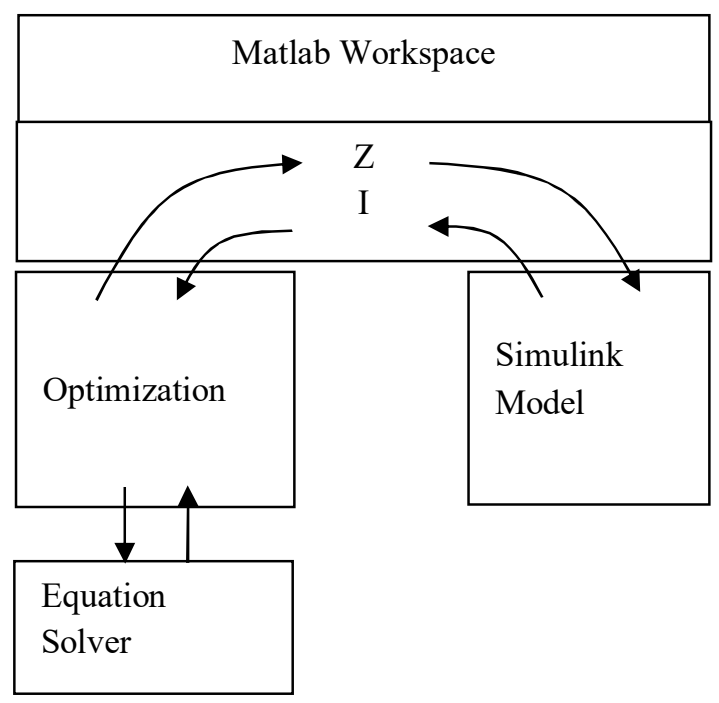

\section{Рис. 5 - Схема взаимодействия между расчетными модулями пакета Matlab}

Взаимодействие между компонентами производится через рабочее пространство Маtlab. Процедура Орtimization присваивает переменной Z значения заданных мощностей, которые считываются в среде моделирования Simulink. Запуск процесса моделирования работы котлов производится встроенной функцией Matlab sim('Model_name'). B cвою очередь, из Simulink в рабочую область сохраняются значения целевой функции I с помощью блока ТоWоrkspace. Для корректной работы поискового метода в модуле расчета целевой функции конечное значение умножается на -1. Таким образом процедура Optimization осуществляет поиск минимума функции.

Графики переходных процессов поиска оптимального распределения нагрузки показаны на рисунке 6.

При моделировании предполагалось, что суммарная нагрузка котлов изменялась от 2,1 до 2,6 единиц максимальной нагрузки. Время поиска минимума целевой функции с учетом динамических характеристик котлов составило 955 с. При этом полное время выполнения поисковой функции без учета ожидания переходного процесса в котлах составляло около 1 минуты.

График изменения значения целевой функции показан на рисунке 7.

Следует также отметить, что при моделировании система (22) решалась численно. Результаты совпали с точностью вычислений в Matlab.

\section{5. Выводы}

В качестве выводов можно отметить следующее: указанный алгоритм поиска можно применять для любого количества работающего оборудования. Однако получить аналитическое решение системы уравнений аналогичной (22) уже затруднительно для четырех котлов, т.к. необходимо решать систему уравнений третьего порядка. Для пяти котлов будет уже система уравнений четвертого порядка. Однако использование численных методов решения позволяет оптимизировать любое количество оборудования.

Производительность средств вычислительной техники и цифровых регуляторов позволяют реализовать указанный поисковый алгоритм в составе современных АСУТП, т.к. длительность переходного процесса регулирования при изменении задания регулятору превышает время расчета на два порядка. 


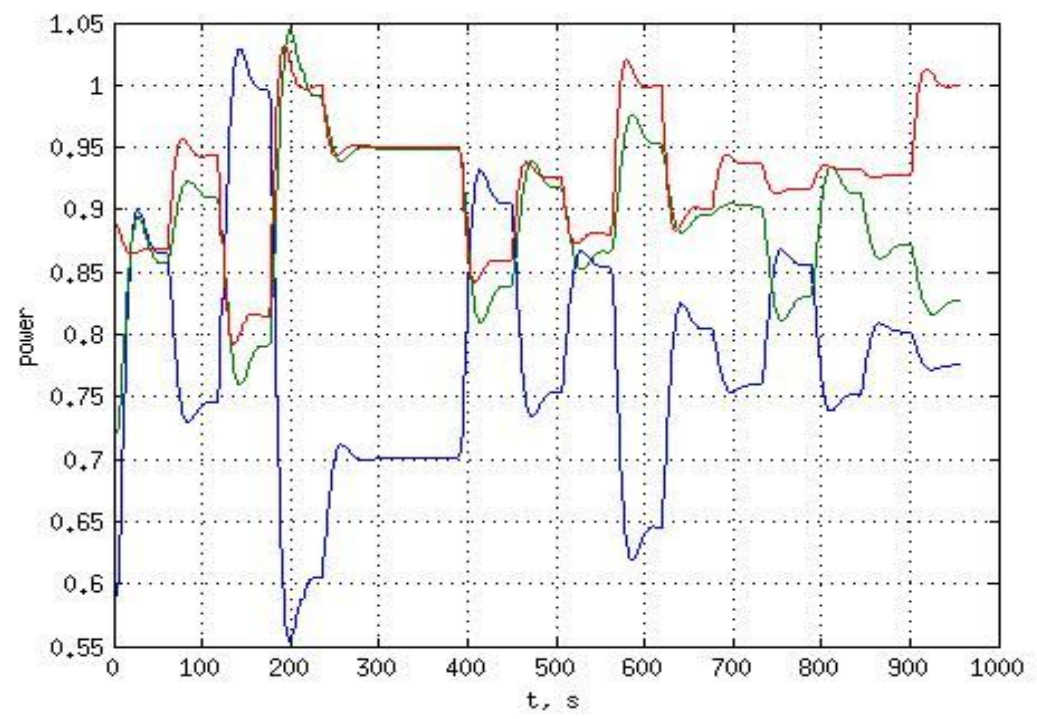

Рис. 6 - Переходные процессы в группе котлов при поиске оптимальной нагрузки

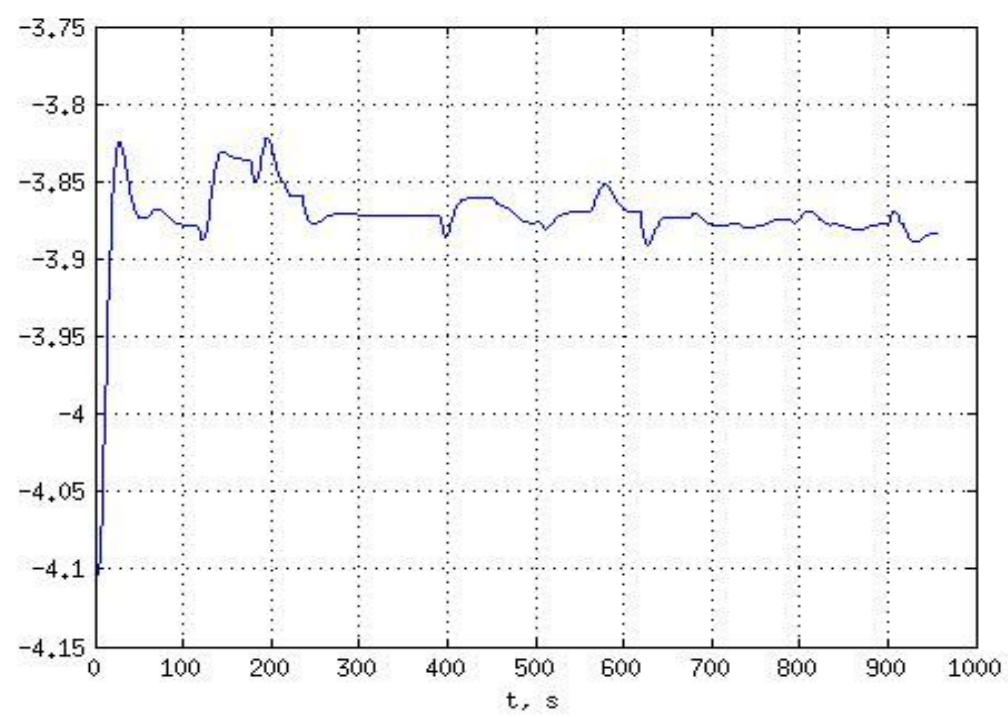

Рис. 7 - Изменение значения целевой функции при изменении нагрузки с 2,4 до 2.6 относительных единиц

Литература

[1] Кожевников Н.Н. Экономика и управление энергетическими предприятиями: Учебник для студ. высш. учеб. заведений / Т. Ф. Басова, Е. И. Борисов, В. В. Бологова и др.; Под ред. Н. Н. Кожевникова. - М.: Издательский центр «Академия», 2004. - 432 с.

[2] Макарчьян В.А. Программный комплекс распределения нагрузок ТЭЦ со сложным составом оборудования, схемами отпуска тепла и электроэнергии / В.А. Макарчьян, А.Н. Черняев, А.В. Андрюшин и др // Теплоэнергетика. 2013. - №5. - С. 71-77.

[3] Реймов К.М. Определение критерия оптимального распределения активной нагрузки между агрегатами ТЭС. // Автоматизированные технологии и производства. - 2016. - № 2 (12). С. 25-27.

[4] Аракелян Э. К. Проблемы современных автоматизированных систем управления технологическим процессом на базе программно-технических комплексов и возможный путь их решения / Э. К. Аракелян, Е. Д. Васильев, С. Р. Хуршудян // Вестник МЭИ. - 2014. - № 1. С. 15-20.

[5] Озерова И. П. Обоснование необходимости распределения нагрузок между агрегатами ТЭС на базе комплексного критерия / И. П. Озерова, Р. С. Новиков // Известия Томского политехнического университета [Известия ТПУ]. - 2002. - Т. 305, вып. 2 : труды II-го семинара вузов Сибири и Дальнего Востока по теплофизике и теплоэнергетике, Томск, 24-25 октября 2001 г.. - С. 108-114. 
[6] Аракелян Э.К. Оптимальное распределение нагрузки между параллельно работающими энергетическими блоками с учетом фактора надежности / Э.К. Аракелян, Н.В. Мань, Н.Ч. Хунг // Вестник МЭИ. 1997. № 3. С. 15-20.

[7] Плетнев Г.П. Автоматическое управление вредными выбросами в переменных режимах ТЭС / Г.П. Плетнев, Т.Е. Щедеркина, А.С. Горбачев //Теплоэнергетика, 1995. № 4. - С. 54-56.

[8] Добровольская Т.С. Определение оптимального алгоритма работы оборудования методом динамического программирования / Т. С. Добровольская // Восточно-Европейский журнал передовых технологий. - 2014. - Т. 5, № 8 (71). - C. 53-58.

[9] Економіка підприємства : Підручник / За заг. ред. С.Ф. Покропивного. - 2-ге вид., перероб. та доп.- К.: КНЕУ, 2000.- 528 с. : іл.

[10] Тепловой расчет котлов. Нормативный метод. Санкт-Петербург: 1998. Изд. 3-е, перераб.

References

[1] N. N. Kozhevnikov. Ekonomika i upravlenie energeticheskimi predpriyatiyami: Uchebnik dlya stud. vyissh. ucheb. zavedeniy. Moscow Izdatelskiy tsentr «Akademiya», 432 p., 2004.

[2] V.A. Makarchyan, Programmnyiy kompleks raspredeleniya nagruzok TETs so slozhnyim sostavom oborudovaniya, shemami otpuska tepla i elektroenergii, Teploenergetika. no. 5, pp. 71-77, 2013.

[3] K.M. Reymov. "Opredelenie kriteriya optimalnogo raspredeleniya aktivnoy nagruzki mezhdu agregatami TES", Avtomatizirovannyie tehnologii i proizvodstva. no. 2 (12), pp. 25-27, 2016.

[4] E. K. Arakelyan i dr. "Problemyi sovremennyih avtomatizirovannyih sistem upravleniya tehnologicheskim protsessom na baze programmno-tehnicheskih kompleksovi vozmozhnyiy put ih resheniya”, Vestnik MEI no 1. pp. 15-20, 2014.

[5] I. P. Ozerova "Obosnovanie neobhodimosti raspredeleniya nagruzok mezhdu agregatami TES na baze kompleksnogo kriteriya", Izvestiya Tomskogo politehnicheskogo universiteta [Izvestiya TPU].: trudyi II-go seminara vuzov Sibiri i Dalnego Vostoka po teplofizike i teploenergetike, Tomsk, 24-25 oktyabrya 2001, 2002 vol. 305 pp. 108-114.

[6] E.K. Arakelyan, N.V. Man and N.Ch. Hung "Optimalnoe raspredelenie nagruzki mezhdu parallelno rabotayuschimi energeticheskimi blokami s uchetom faktora nadezhnosti”, Vestnik MEI. no. 3, pp. 15-20, 1997.

[7] G.P. Pletnev, T.E. Schederkina and A.C. Gorbachev "Avtomaticheskoe upravlenie vrednyimi vyibrosami v peremennyih rezhimah TES”, Teploenergetika, no. 4, pp. 54-56, 1995.

[8] T.S. Dobrovolskaya "Opredelenie optimalnogo algoritma rabotyi oborudovaniya metodom dinamicheskogo programmirovaniya", Vostochno-Evropeyskiy zhurnal peredovyih tehnologiy. vol. 5, no. 8 (71), pp. 53-58,-2014.

[9] S.F. Pokropivniy. Ekonomika pidpryemstva : Pidruchnik 2nd ed. Kiev : KNEU, 2000. - 528 p.

[10] Teplovoy raschet kotlov. Normativnyiy metod. 3rd ed. Sankt-Peterburg 1998.

УДК 004.435

\title{
ПАРАДИГМА ПОДАННЯ ЛІНГВІСТИЧНОГО ЗАБЕЗПЕЧЕННЯ ЗА ДОПОМОГОЮ ПОРОДЖУВАЛЬНИХ ГРАМАТИК
}

\author{
С. Великодний ${ }^{1}$ О. Тимофєєва ${ }^{2}$ \\ ${ }^{1}$ Національний університет «Одеська морська академія», ${ }^{2}$ Одеський державний екологічний університет \\ E-mail: ${ }^{1}$ velykodniy@gmail.com; ${ }^{2}$ hellena1985th@gmail.com
}

Copyright $(\subseteq 2017$ by author and the journal "Automation technological and business - processes".

This work is licensed under the Creative Commons Attribution International License (CC BY).

http://creativecommons.org/licenses/by/4.0/

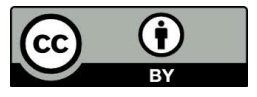

ONAFT

Open Access

Анотація: В статті розглянуто створення системи понять, щзо формують парадигму реінжинірингу інформаційних технологій, який необхідний у випадку їх еволюиійного розвитку. Лінгвістичне забезпечення 